\title{
INDICADORES BIBLIOMÉTRICOS SOBRE EDUCAÇÃO EM ENGENHARIA EM DIFERENTES BASES DE DADOS
}

Mariana S. Lopes - lopes_ms@outlook.com

Programa de Pós-Graduação em Ciência e Engenharia de Materiais (PPGCEM) - UFSCar

Núcleo de Informação Tecnológica em Materiais (NIT) - UFSCar

Rodovia Washington Luís, km 235

13565905 - São Carlos - São Paulo

Humberto D. de Almeida Filho - humbertodaf@ outlook.com

Daniel R. Leiva - daniel.leiva@ufscar.br

Departamento de Engenharia de Materiais (DEMa) - UFSCar

Programa de Pós-Graduação em Ciência e Engenharia de Materiais (PPGCEM) - UFSCar

Núcleo de Informação Tecnológica em Materiais (NIT) - UFSCar

Rodovia Washington Luís, km 235

13565905 - São Carlos - São Paulo

Resumo: Buscando atender à necessidade do mercado por profissionais competentes, não só na área técnica, mas também com habilidades de liderança, empreendedorismo e inovação, surgem esforços ao redor do mundo para modernizar os cursos de engenharia, dando cada vez. mais impulso ao campo de pesquisa em Educação em Engenharia. Uma das formas de avaliar o impacto da pesquisa cientifica e analisar seus resultados é através do uso de indicadores de ciência e tecnologia. Este trabalho se propôs a elaborar indicadores bibliométricos sobre educação em engenharia a partir das pesquisas disponibilizadas na Web of Science e Scopus nos últimos 20 anos (2000 a 2019). Foram analisados: número de publicações por ano; países, instituições e autores mais relevantes e tendências de pesquisa na área. Após a análise dos dados foi possivel constatar a hegemonia dos EUA nas pesquisas em educação em engenharia através do número de publicações, principais instituições e autores. O Brasil não se destacou na área, sendo responsável por apenas uma pequena parcela das publicações analisadas, embora políticas públicas implantadas recentemente venham tentando gerar melhorias no panorama atual. Além disso, foram identificadas tendências de pesquisas em metodologias ativas de aprendizagem e uso de ferramentas computacionais de ensino, sendo necessários estudos futuros mais aprofundados para estabelecer o estado da arte destes temas, as redes de colaboração entre países, instituições e autores e uma projeção de crescimento da área.

Palavras-chave: Educação em engenharia. Indicadores bibliométricos. Bases de dados. Bibliometria. 


\section{INTRODUÇÃO}

A Engenharia está diretamente relacionada ao desenvolvimento industrial e socioeconômico de qualquer país, devido à sua capacidade intrínseca de absorver e gerar novas tecnologias, sendo, portanto, uma área estratégica. Buscando atender à necessidade do mercado por profissionais competentes, não só na área técnica, mas também com habilidades de liderança, empreendedorismo e inovação, surgem esforços ao redor do mundo para modernizar os cursos de engenharia, dando cada vez mais impulso ao campo de pesquisa em Educação em Engenharia. Deste modo, torna-se importante um maior entendimento da área, bem como; a maneira como essas pesquisas estão apresentadas nas bases de dados, permitindo avaliar a disponibilidade e abrangência de informações pertinentes para consulta e emprego na melhoria do ensino (IEDI, 2010; LUCENA et al., 2008; OLIVEIRA; PINTO, 2006).

Uma das formas de avaliar o impacto da pesquisa cientifica e analisar seus resultados é através do uso de indicadores de ciência e tecnologia. A bibliometria é uma ferramenta importante para quantificar as pesquisas científicas existentes e fornecer um panorama atual e comparativo entre o Brasil e o mundo (ALVES; DE FARIA; DO AMARAL, 2017; HOFFMANN et al., 2005).

Os intitulados indicadores científicos e bibliométricos têm atraído cada vez mais interesse da comunidade científica, devido ao seu uso crescente como elemento de suporte à avaliação de produtividade e aos processos de estabelecimento de políticas de fomento que encabeçam a alocação de recursos (PINTO; MATIAS, 2011).

Levando em consideração as informações mencionadas, este trabalho se propôs a elaborar indicadores acerca da pesquisa científica relacionada à educação em engenharia, presente em duas das principais bases de dados atualmente, a Web of Science e a Scopus. Foram analisados: número de publicações por ano; países, instituições e autores mais relevantes; diversidade de gênero entre os pesquisadores e tendências de pesquisa na área.

\section{METODOLOGIA}

Os critérios de seleção das bases de dados a serem utilizadas neste trabalho foram multidisciplinaridade, alcance internacional, fácil acesso e interface amigável do buscador. Foi definido para análise o período dos últimos 20 anos (2000 a 2019), de forma a garantir que os resultados obtidos fossem suficientes para traçar tanto o panorama atual, quanto à cronologia $\mathrm{e}$ à dinâmica das pesquisas na área.

Ao realizar uma busca por Educação em Engenharia no portal de periódicos da CAPES/MEC, utilizando o mecanismo de busca avançada para pesquisar pela expressão "engineering education" em publicações dos últimos 20 anos, foram obtidos 38.272 resultados. A maior parte das publicações encontradas é proveniente das bases de dados Scopus, da Editora Elsevier (18.340 resultados) e Web of Science (WoS), da Editora Thomson Reuters (14.240 resultados). Além disso, ambas as bases são multidisciplinares, indexando milhares de periódicos de diversos países, possuindo um grande alcance, atendendo, portanto, aos critérios mencionados anteriormente e constituindo uma escolha adequada à realização deste trabalho.

Uma vez escolhidas as bases de dados a serem utilizadas, foi necessário definir a expressão de busca que melhor se adequasse a cada base, devido às diferenças entre os mecanismos de busca disponíveis, como campos de busca, filtros e códigos, sendo necessário um breve estudo sobre as particularidades de cada base e uma iteração inicial para teste.

Avaliando os resultados iniciais, percebeu-se que ao pesquisar a expressão de busca nos tópicos: título, resumo, assunto e palavras-chave; em ambas as bases muitos periódicos não 
relacionados ao tema foram encontrados. Isso ocorre, pois, as bases utilizam algoritmos para definir as palavras-chave dos artigos automaticamente, a partir de sua repetição no corpo do texto, mas nem sempre uma palavra utilizada muitas vezes representa a ideia central abordada, gerando resultados que não condizem com o tema buscado. Foi necessária, portanto, repetir a busca removendo o campo "palavras-chave" dos tópicos pesquisados, para filtrar melhor os resultados.

Na base WoS (Tabela 1), foi necessária ainda uma terceira busca para que os resultados fossem refinados, excluindo as categorias da própria base não relacionadas ao tema de pesquisa, como "PHARMACOLOGY", "OCEANOGRAPHY” e "HORTICULTURE".

Tabela 1 - Expressão de busca e número de resultados para o período de 2000 a 2019, utilizando a base de dados Web of Science.

\begin{tabular}{ll} 
Expressão de busca & Resultados \\
\hline $\mathrm{TS}=$ ("engineering education") & 12.330 \\
\hline $\mathrm{TI}=$ ("engineering education") OR & \\
$\mathrm{AB}=$ ("engineering education") OR & 8.844 \\
$\mathrm{SU}=$ ("engineering education") & \\
\hline Excluindo as categorias não relacionadas ao tema & 6.585 \\
\hline
\end{tabular}

Fonte: Os autores, 2020.

Enquanto que, na base Scopus (Tabela 2), foi necessária a exclusão de assuntos como "Neuroscience", "Pharmacology, Toxicology and Pharmaceutics" e "Immunology and Microbiology" e expressões como "High School Students", "Computer Hardware" e "Budget Control" para limitar a pesquisa aos registros adequados ao tema.

Tabela 2 - Expressão de busca e número de resultados para o período de 2000 a 2019, utilizando a base de dados Scopus.

\begin{tabular}{ll} 
Expressão de busca & Resultados \\
\hline TITLE-ABS-KEY (\{engineering education $\})$ & 83.506 \\
AND PUBYEAR $>1999$ AND PUBYEAR $<2020$ & 30.232 \\
\hline TITLE-ABS $(\{$ engineering education $\})$ & \\
AND PUBYEAR $>1999$ AND PUBYEAR $<2020$ & 22.027 \\
\hline Excluindo os temas e palavras-chaves não relacionadas ao tema &
\end{tabular}

Ambas as bases apresentam limitações para a exportação dos resultados. Na base $W o S$ é possível exportar apenas 500 registros por vez, em diversos formatos, sendo escolhido para este trabalho o "registro completo e referências citadas" no formato "texto sem formatação". Portanto, foi realizada a exportação dos registros em frações adequadamente nomeadas e agrupadas em uma pasta, para posterior importação.

Já na base Scopus, é possível exportar até 2.000 registros de forma detalhada (informações bibliográficas, de citação, resumo, palavras chave, financiamento, entre outras) em diversos formatos e até 20.000 registros, no formato CSV (Excel), contendo apenas informações de citação. Como o intuito era obter informações detalhadas dos registros, foi necessário então, fracionar a pesquisa em períodos menores de tempo, para obter grupos contendo menos de 2 mil registros, para exportação.

Uma vez obtidos todos os registros desejados, foi realizada a importação para o software VantagePoint v5.0, gerando um arquivo para cada base de dados, nos quais foi realizado o tratamento dos dados e construção dos indicadores bibliométricos. Posteriormente, foi utilizado 
o software de planilhas eletrônicas Microsoft Excel para a junção dos resultados e construção dos gráficos apresentados na seção a seguir.

\section{RESULTADOS E DISCUSSÃO}

Iniciando a análise dos resultados, os dados a respeito do número anual de publicações em educação em engenharia dos últimos 20 anos estão apresentados na Figura 1.

Figura 1 - Número de publicações por ano, nos últimos 20 anos (2000-2019).

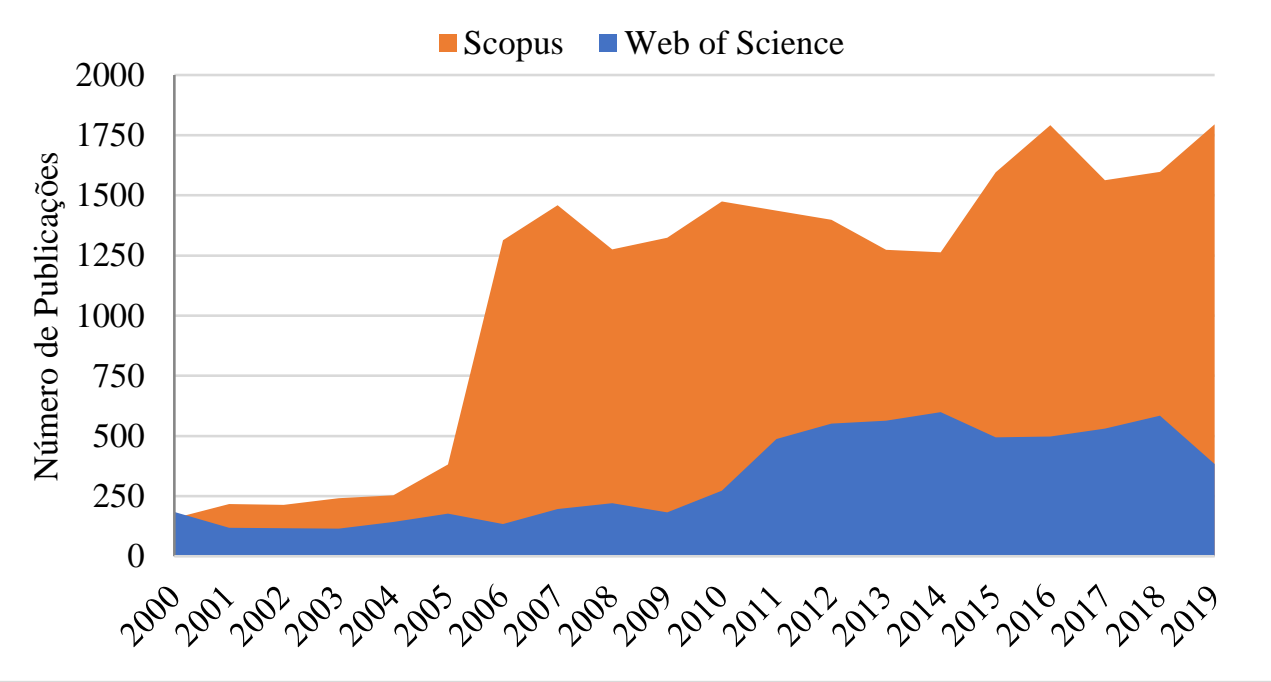

Fonte: Os autores, 2020.

Pode-se observar que, entre os anos de 2000 a 2005, para a base Scopus, o número total de publicações se manteve estável, sofrendo, porém, em 2006 um aumento considerável neste valor. Após este crescimento em 2006, o resultado no número total de publicações ficou mais uma vez estável até que, em 2015, sofreu um novo aumento significativo. Já para a base de dados WoS, os valores continuaram estáveis até o ano de 2011, quando também houve um acréscimo significativo em seus valores.

Em uma busca mais aprofundada, na tentativa de justificar algumas das tendências observadas, verificou-se que, entre os anos de 2011 a 2014, os anais do congresso American Society for Engineering Education (ASEE) foram indexados à WoS. Por se tratar de um evento de grandes proporções, com a presença de uma grande quantidade de pesquisadores, foi gerado um alto número de artigos científicos na área, justificando o crescimento observado. Entretanto, para a base Scopus não foram encontrados dados que justificassem o grande aumento ocorrido em 2006 (MARCOS; LEIVA, 2019).

Quanto ao número de publicações por país (Figura 2), analisando os dados da base $W o S$, é possível observar a dominância dos Estados Unidos da América (EUA), contribuindo com $35,95 \%$ das publicações (2.367 publicações), mais do que o dobro de publicações da China (555 publicações), segundo país com maior número de publicações. Em seguida, temos contribuições significativas da Espanha (338 publicações), Austrália (283 publicações) e Alemanha (218 publicações), completando o grupo dos cinco países com maior número de publicações, além de serem responsáveis por mais da metade $(57,11 \%)$ do total de publicações na área, para o período analisado. 
Figura 2 - Número de publicações por país, para o período de 2000 a 2019.

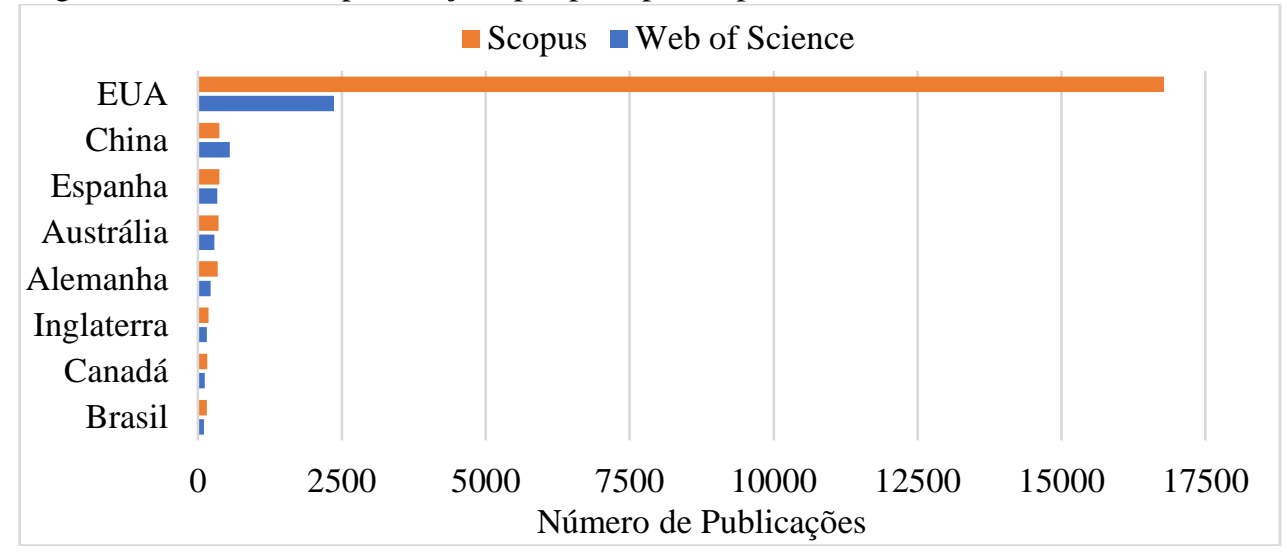

Fonte: Os autores, 2020.

Na base Scopus, a predominância dos EUA é ainda maior, detendo 76,17\% das publicações (16.779 publicações), sendo, portanto, o país com maior número de publicações em ambas as bases estudadas. Enquanto o Canadá, segundo país com mais publicações na base, conta com apenas $1,70 \%$ das publicações (375 publicações), seguido bem de perto pela China com 1,69\% (372 publicações); Inglaterra com 1,64\% (362 publicações) e Austrália com 1,56\% (344 publicações).

O Brasil foi inserido na figura apenas para fins expositivos, sendo responsável por somente $1,55 \%$ das publicações (102 publicações) da plataforma WoS e 0,64\% (141 publicações) da Scopus, no período analisado, não constituindo, deste modo, um país de destaque na área de educação em engenharia. No entanto, algumas políticas públicas implantadas recentemente podem gerar melhorias no panorama atual. Está em vigência, desde 2019, o "Programa BrasilEstados Unidos de Modernização da Educação Superior na Graduação (PMG-EUA)”, voltado aos cursos de engenharia, cujos objetivos principais são incentivar a criatividade, inovação e empreendedorismo aliados à uma forte base teórica; criação de currículos, metodologias de ensino e gestão de cursos que possam ser aplicados à realidade dos cursos superiores brasileiros e a melhoria da educação e adequação às tendências mundiais da Engenharia a partir da criação de redes de colaboração entre o Brasil e os EUA. Foram também aprovadas novas diretrizes curriculares nacionais para os cursos de engenharia com foco na formação a partir da construção de competências, definição do perfil desejável do egresso em engenharia, utilização de metodologias ativas de ensino e aprendizagem e flexibilização curricular (CAPES, 2018; MINISTÉRIO DA EDUCAÇÃO, 2019).

Cabe mencionar ainda que uma publicação é atribuída a um país, quando ao menos um de seus autores apresenta a nacionalidade referida, podendo, portanto, uma mesma publicação ser atribuída à vários países diferentes, de acordo com a nacionalidade de seus autores.

A dominância dos EUA na área de educação em engenharia se mantém também em relação ao número de publicações por instituição de ensino, como pode ser visto na Figura 3. 
Figura 3 - Número de publicações por instituição de ensino, para o período de 2000 a 2019.

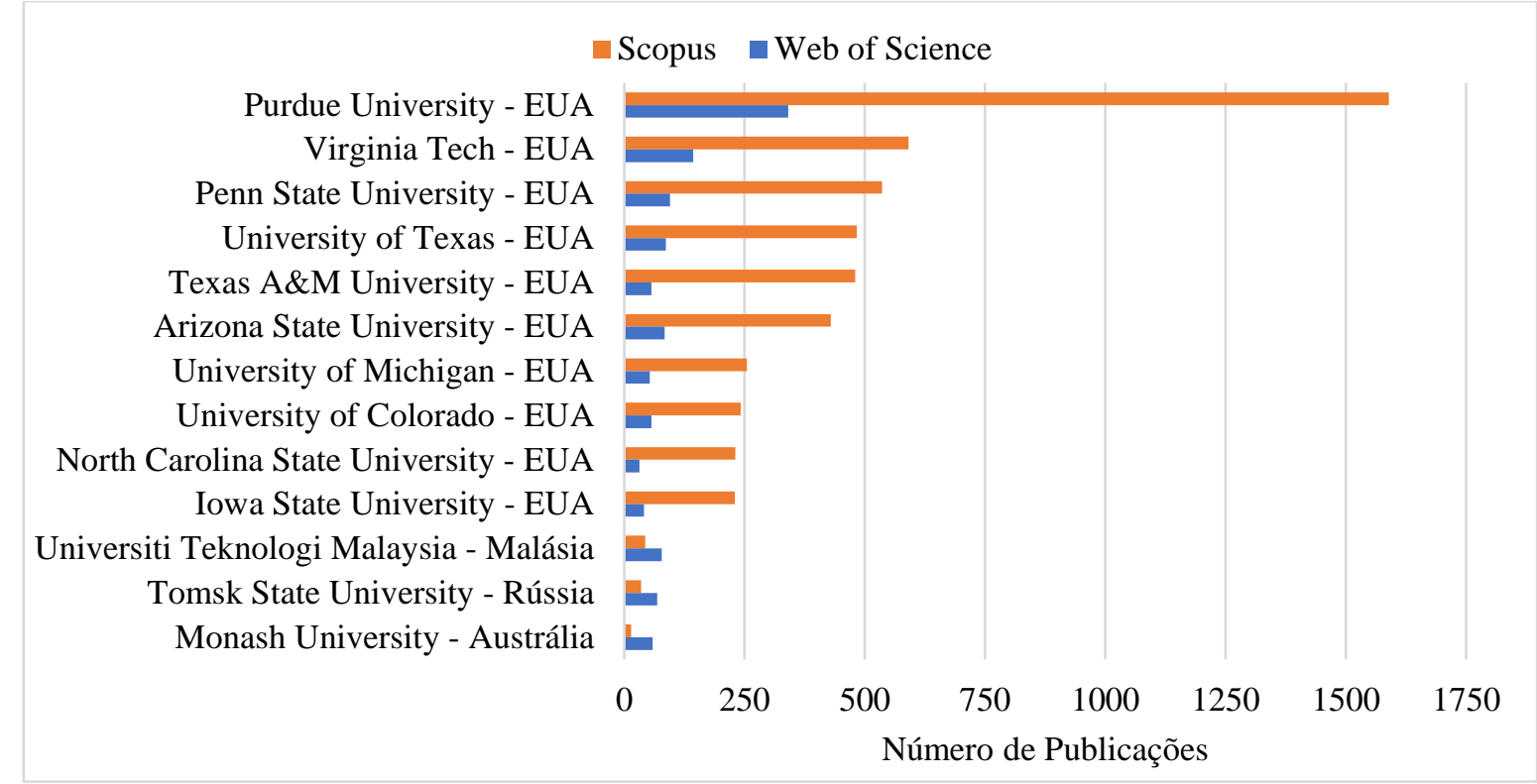

Fonte: Os autores, 2020.

É possível observar que, entre as dez instituições com maior número de publicações na Scopus, todas estão localizadas nos EUA e entre as dez instituições com mais publicações na $W o S$, apenas três instituições localizam-se em outros países.

Nas duas bases, o maior número de publicações foi atribuído à Purdue University (1589 publicações na Scopus e 341 na WoS), seguido pela Virginia Tech (591 publicações na Scopus e 143 na WoS), Penn State University (536 publicações na Scopus e 95 na WoS) e University of Texas (483 publicações na Scopus e 86 na WoS). Cabe mencionar que a Purdue University, a Virginia Tech e a Arizona State University possuem departamentos exclusivamente dedicados à educação em engenharia, além de contar com programas de pós-graduação específicos na área, justificando assim seu alto número de publicações (BENSON et al., 2010).

A Figura 4 nos apresenta um panorama geral sobre autores com maior número de publicações na área em cada plataforma. 
Figura 4 - Número de publicações por autor no período de 2000 a 2019 e país de vinculação atual.

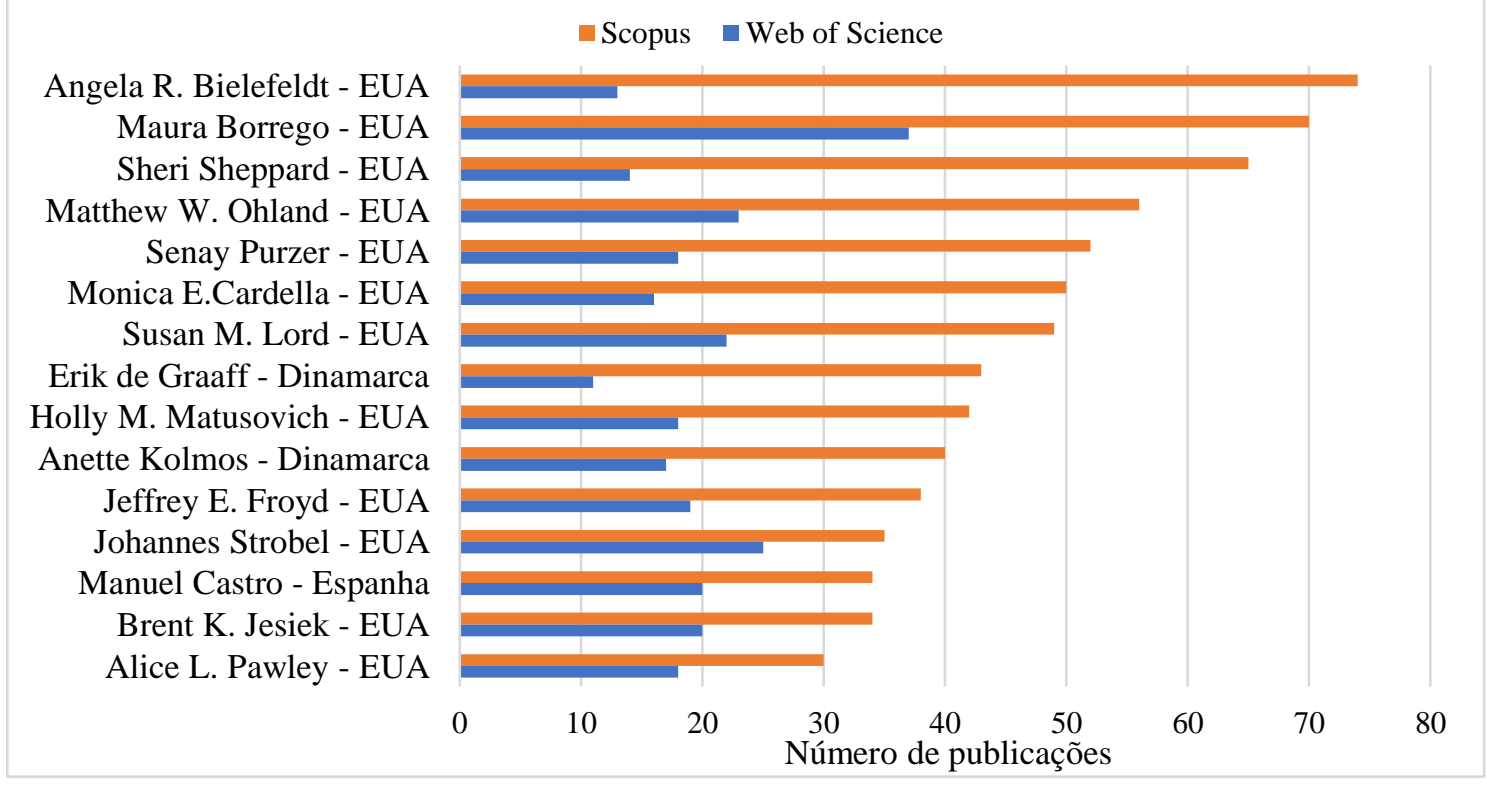

Fonte: Os autores, 2020.

Podemos notar que, entre os quinze autores com maior número de publicações, apenas três autores não atuam nos EUA e nove são mulheres, indicando a representatividade feminina na área. Além disso, a Purdue University é a instituição com mais autores em destaque (cinco dos quinze autores mencionados).

Para um maior entendimento a respeitos dos assuntos mais estudados pela área da educação em engenharia, foi realizado o levantamento das palavras-chave que mais vezes apareceram nas publicações englobadas pela pesquisa. Os resultados estão apresentados na Figura 5.

Figura 5 - Palavras-chave de maior ocorrência.

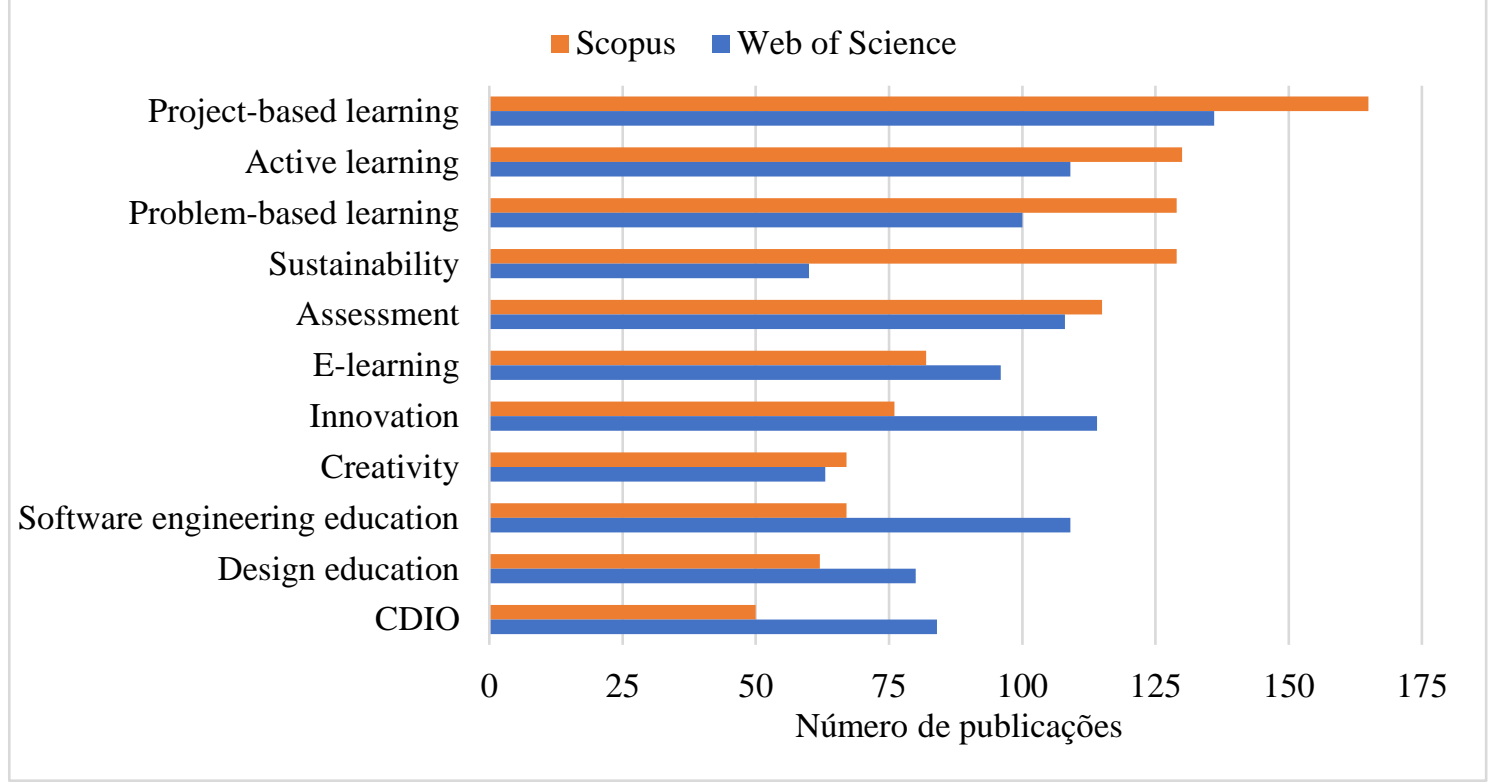

Fonte: Os autores, 2020. 
Apesar da ocorrência de termos genéricos, como "Sustainability", "Assessment", "Innovation" e "Creativity", que retirados de contexto não permitem definir exatamente qual o tema de pesquisa, observa-se a tendência por pesquisas sobre metodologias ativas de ensino e aprendizagem, através da grande ocorrência dos termos "Project-Based Learning", "Active Learning", "Problem-Based Learning" e "CDIO" (Conceive, Design, Implement, Operate), que tem como objetivo central definir "como o estudante pode aprender mais e melhor", colocandoo, desta forma, como protagonista no desenvolvimento das competências necessárias para o mercado e para a academia. Houve também a recorrência de termos referentes ao uso de ferramentas computacionais no ensino, através dos termos "E-learning" e "Software engineering education", área que tende a crescer em decorrência da COVID-19, tendo em vista que nos últimos meses tivemos mudanças globais no ensino, fazendo com que grande parte das instituições optasse pelo sistema de ensino remoto como medida de proteção aos alunos e docentes (FERREIRA et al., 2018).

\section{CONCLUSÃO}

O trabalho realizado teve como finalidade obter uma visão geral acerca da situação e direcionamentos sobre as pesquisas em educação em engenharia nas bases de dados Web of Science e Scopus, tornando possível uma melhor contextualização da produção científica na área em uma esfera mundial, identificando possíveis tendências, os principais autores e instituições atuantes na área.

Podemos ressaltar a grande notoriedade dos EUA nessa temática de estudo, sendo o país líder em número de publicações em ambas as bases de dados durante o período analisado. Essa tendência se mantém em relação às instituições com maior número de publicações. Na Scopus, por exemplo, todas as dez principais instituições com mais publicações são dos EUA e, na $W o S$, apenas três instituições localizam-se em outros países. Vale ressaltar também que, as instituições que lideram tais publicações ofertam programas de pós-graduação específicos na área, além da disponibilidade de departamentos dedicados exclusivamente à educação em engenharia. Além disso, entre os quinze autores com maior número de publicações, apenas três não lecionam nos EUA.

É importante mencionar ainda que, dos quinze autores mencionados anteriormente, a maioria são mulheres (nove mulheres e seis homens), sendo inclusive as responsáveis pelos três maiores números de publicações, indicando uma significativa representatividade feminina na área.

Já se tratando do âmbito nacional, o Brasil é responsável por apenas uma pequena parcela das publicações analisadas, não constituindo, deste modo, um país de destaque na área de educação em engenharia, mas que apresenta possibilidade de crescimento nos próximos anos devido às políticas de modernização do ensino implantadas recentemente.

Quanto à análise das palavras-chave que mais se repetem, notou-se uma grande ocorrência de termos ligados ao uso de metodologias ativas de aprendizagem e de ferramentas computacionais no ensino, o que faz todo o sentido no contexto de uma sociedade tecnológica, em que torna-se necessário criar formas mais estimulantes de aprendizagem para manter a atenção das novas gerações, como o uso de softwares para visualização de conteúdos complexos e resolução de problemas que permitam o discente traçar paralelos entre o conteúdo e a prática.

Uma proposta interessante para trabalhos futuros está na análise de dados de um período maior de tempo, permitindo traçar uma projeção para o crescimento da área com base em seu comportamento ao longo dos anos, bem como, a comparação dos dados de outras bases e uma melhor utilização dos filtros disponíveis. Outro caminho a ser seguido é a realização de uma 
análise mais aprofundada dos temas pesquisados na área, de forma a estabelecer seu estado da arte, tal como as redes de colaboração entre países, instituições e autores, traçando um panorama sobre quais são e onde estas pesquisas estão concentradas.

\section{Agradecimentos}

O presente trabalho foi realizado com apoio da Coordenação de Aperfeiçoamento de Pessoal de Nível Superior - Brasil (CAPES) - Código de Financiamento 001.

\section{REFERÊNCIAS}

ALVES, M. C.; DE FARIA, L. I. L.; DO AMARAL, R. M. Visualização de informação para simplificar o entendimento de indicadores sobre avaliação da ciência e tecnologia. RDBCI: Revista Digital de Biblioteconomia e Ciência da Informação, v. 15, n. 2, p. 324-348, 17 abr. 2017.

BENSON, L. C. et al. Engineering Education: Departments, Degrees and Directions. The International Journal of Engineering Education, v. 26, n. 5, p. 7, 2010.

CAPES - Coordenação de Aperfeiçoamento de Pessoal de Nível Superior. EDITAL n ${ }^{\circ}$ 23/2018: "Programa Brasil-Estados Unidos de modernização da educação superior na graduação (PMG-EUA)”. Brasília: CAPES/CNE/COMISSÃ̃ FULBRIGHT, 2018. Disponível em: https://www.capes.gov.br/images/stories/download/editais/11062018Edital_23_PMG_EUA2.pdf. Acesso em: 27 jun. 2020.

FERREIRA, M. et al. METODOLOGIAS ATIVAS DE APRENDIZAGEM APLICADAS NO ENSINO DA ENGENHARIA. In: CIET:EnPED, 4., 2018, São Carlos. Anais [...]. São Carlos: Secretaria Geral de Educação a Distância - Universidade Federal de São Carlos, 2018. Disponível em: https://cietenped.ufscar.br/submissao/index.php/2018/article/view/877. Acesso em: 27 mai. 2020.

HOFFMANN, W. A. M. et al. Análise da Produção Científica a partir de Indicadores Bibliométricos. In: GREGOLIN, J. Â. R. (coord.). Indicadores de ciência, tecnologia e inovação em São Paulo 2004. São Paulo: FAPESP, 2005. v. 1, cap. 5, p. 5-43.

IEDI - Instituto de Estudos para o Desenvolvimento Industrial. A Formação de Engenheiros no Brasil: Desafio ao Crescimento e à Inovação. Carta IEDI n. 424, São Paulo, 16 jul. 2010. Carta. Disponível em:

https://iedi.org.br/cartas/carta_iedi_n_424_a_formacao_de_engenheiros_no_brasil_desafio_a o_crescimento_e_a_inovacao.html. Acesso em: 16 mai. 2020.

LUCENA, J. et al. Competencies Beyond Countries: The Re-Organization of Engineering Education in the United States, Europe, and Latin America. Journal of Engineering Education, v. 97, n. 4, p. 433-447, out. 2008.

MARCOS, L. P.; LEIVA, D. R. Indicadores Bibliométricos de Publicações sobre Educação em Engenharia. In: CONGRESSO BRASILEIRO DE EDUCAÇÃO EM ENGENHARIA, 47., 2019, Fortaleza. Anais [...]. Fortaleza: Associação Brasileira de Educação em 
Engenharia, 2019. Disponível em:

http://www.abenge.org.br/sis_submetidos.php?acao=abrir\&evento=COBENGE19\&codigo=C OBENGE19_00126_00002313.pdf. Acesso em: 23 mai. 2020.

BRASIL, Ministério da Educação PROCESSO No: 23001.000141/2015-11. Diretrizes Curriculares Nacionais do Curso de Graduação em Engenharia. Diário Oficial da União: seção 1, Brasília, DF, p. 109, 23 abr. 2019.

OLIVEIRA, V. F. DE; PINTO, D. P. Educação em Engenharia como Área do Conhecimento. In: CONGRESSO BRASILEIRO DE ENSINO DE ENGENHARIA, 34., Passo Fundo. Anais [...]. Passo Fundo: Associação Brasileira de Educação em Engenharia, 2006. Disponível em: http://www.abenge.org.br/cobenge/arquivos/13/artigos/12_85_357.pdf. Acesso em: 14 mai. 2020.

PINTO, A. L.; MATIAS, M. Indicadores Científicos e as Universidades Brasileiras. Informação \& Informação, v. 16, n. 3, p. 1-18, 24 dez. 2011.

\title{
BIBLIOMETRIC INDICATORS ON ENGINEERING EDUCATION IN DIFFERENT DATABASES
}

\begin{abstract}
Looking for attend the market needs for competent professionals, not only in the technical area, but also with leadership skills, entrepreneurship and innovation, efforts are emerging around the world to modernize engineering courses, giving impulse to the field of research on Engineering Education. One of the ways to assess the impact of scientific research and analyze its results is through the use of science and technology indicators. This work aimed to develop bibliometric indicators on engineering education based on the research available on the Web of Science and Scopus in the last 20 years (2000 to 2019). Were analyzed: number of publications per year; the most relevant countries, institutions and authors and research trends in the area. After analyzing the data, it was possible to verify the USA hegemony in engineering education research's through the number of publications, main institutions and authors. Brazil, however, did not stand out in the area, being responsible for only a small portion of the analyzed publications, although public policies recently implemented have been trying to generate improvements in the current scenario. In addition, research trends were identified in active learning methodologies and the use of computational teaching tools, requiring further in-depth studies to establish the state of the art of these themes, collaboration networks between countries, institutions and authors and a projection of the area growth.
\end{abstract}

Keywords: Engineering Education. Bibliometric Indicators. Data Base. Bibliometrics. 